\title{
Member heterogenicity and its impacts in wine cooperatives
}

\author{
K. Kuhn, S. Weiss, and J.H. Hanf \\ Hochschule Geisenheim University, von-Lade-Str. 1, 65366 Geisenheim, Germany
}

\begin{abstract}
In 2016, 43000 members of 159 wine cooperatives were growing wine on about $30 \%$ of the total German viticultural area. Those members have different origin, belong to different age groups, their business dispose over different sizes and business models, and all members have individual desires and interests. Thus, the task of the cooperative management and boards is to align the resulting heterogeneous individual aims and interests with the overall aims and interests of the cooperative. Hence the aim of the paper is to answer the question: How the heterogenous member interests can be aligned within the cooperative? In order to answer this question four in-depth expert interviews have been conducted.
\end{abstract}

\section{Einleitung}

Der Zweck deutscher Genossenschaften wird im ersten Paragraph des Genossenschaftsgesetzes als "Gesellschaften von nicht geschlossener Mitgliederzahl, deren Zweck darauf gerichtet ist, den Erwerb oder die Wirtschaft ihrer Mitglieder oder deren soziale oder kulturelle Belange durch gemeinschaftlichen Geschäftsbetrieb zu fördern" (BMJV 2018) beschrieben. Während die wirtschaftliche Förderung der Mitglieder, im Falle von Winzergenossenschaften, die Maximierung der Traubengelder darstellt, kann für soziale und kulturelle Belange keine allgemeingültige Antwort gegeben werden. Wie auch? 2016 bewirtschafteten 43.000 Mitglieder aus 159 weinwirtschaftlichen Genossenschaften rund 30\% der deutschen Rebflächen (vgl. DRV 2017). 43.000 Mitglieder unterschiedlicher Herkünfte, Altersgruppen, Betriebsstrukturen, Interessen und Wünschen. Somit sehen sich die Geschäftsführer und Vorstände mit der Aufgabe befasst, die individuellen Interessen und Ziele der durchschnittlich 270 Genossen in Einklang mit der Ausrichtung der Gesamtheit, also der Genossenschaft zu bringen. Diese Schwierigkeit erkennt auch der Vorsitzende Konrad Gippert der Niederrimsinger Winzergenossenschaft und erklärt, dass der Genossenschaftsgedanke oft nur präsent sei, solange es allen Mitglieder gut gehe. Um dieses Ziel $\mathrm{zu}$ erreichen, müssten jedoch alle Genossen an einem Strick ziehen - und zwar in die gleiche Richtung (vgl. Kricheldorff 2010, S. 1).

Gipperts Metapher umschreibt bereits die grundlegende Fragestellung dieser Arbeit, welche in dieser Arbeit beantwortet werden soll: Wie lassen sich die unterschiedlichen Interessen und Ziele der Mitglieder innerhalb einer Genossenschaft vereinen? Ist dies überhaupt möglich, und welche Auswirkungen hat dies möglicherweise auf die wirtschaftliche Stellung der Genossenschaft?

Um Antworten auf diese Fragen zu finden, werden vier Experteninterviews mit Geschäftsführern württembergischer Winzergenossenschaften durchgeführt und ausgewertet.

\section{Merkmale und auswirkungen der heterogenität}

Aufgrund der langen Historie von Genossenschaften, dem Prinzip der offenen Mitgliedschaften, sowie die zunehmende Konzentrierung durch Fusionen und Aufkäufen lassen sich fünf Merkmale für das Maß an Heterogenität in einer Winzergenossenschaft definieren, dargestellt in Tabelle 1. Weiterhin wird davon ausgegangen, dass sich jedes dieser Heterogenitätsmerkmale auf eine Genossenschaft auswirken kann, sowohl im positiven als auch negativen Sinne. Diese Auswirkungen werden in Tabelle 2 zusammengefasst.

\section{Methodik und durchführung der befragungen}

Die dargelegten Merkmale und Auswirkungen bilden die Grundlage für Experteninterviews mit Geschäftsführern von Genossenschaften. Die Interviews sollen einen Eindruck verschaffen, ob die einzelnen Merkmale überhaupt, und wenn, in welcher Schwere, sich auf eine Genossenschaft auswirken.

Die Auswahl der befragten Genossenschaften basiert auf einer systematischen Stichprobe um ein Minimum an Einflüssen durch unterschiedliche exogene Faktoren sicherzustellen. Per Zufall wurde ein Punkt innerhalb Württembergs gewählt und mit einem ansteigenden Radius, jedoch maximal 50 Kilometer, nach vier Winzergenossenschaften gesucht. Mit diesem Prinzip wurde sichergestellt, dass die befragten Winzergenossenschaften in einem hochgradig homogenen Umfeld wirtschaften.

\section{Ergebnisse der experteninterviews}

\subsection{Alter des mitglieds}

Alle Genossenschaften verzeichnen einen steigenden Altersdurschnitt ihrer Mitglieder. Verschiedene Anreize werden den Jungwinzern geboten, um die Genossenschaft 
Tabelle 1. Merkmale und Aspekte der Heterogenität.

\begin{tabular}{|l|l|}
\hline Merkmal & Aspekte \\
\hline Alter des Mitglieds & $\begin{array}{l}\text { Altersstruktur und } \\
\text { Divergenz }\end{array}$ \\
\hline $\begin{array}{l}\text { Berufserfahrung des } \\
\text { Mitglieds }\end{array}$ & $\begin{array}{l}\text { Ausbildungsniveau und } \\
\text { Berufserfahrung }\end{array}$ \\
\hline Ziele des Mitglieds & $\begin{array}{l}\text { Qualitäts - und } \\
\text { Mengenvorstellungen, } \\
\text { Unternehmensphilosophie }\end{array}$ \\
\hline $\begin{array}{l}\text { Art des } \\
\text { Mitgliedsbetriebs }\end{array}$ & $\begin{array}{l}\text { Vollerwerb, Nebenerwerb, } \\
\text { Hobby und deren } \\
\text { Größenaufteilung, Größe }\end{array}$ \\
\hline $\begin{array}{l}\text { Perspektivische } \\
\text { Ausrichtung des Betriebs }\end{array}$ & Nachfolgeregelung \\
\hline
\end{tabular}

Quelle: Eigene Ermittlung.

Tabelle 2. Auswirkungen der Heterogenität.

\begin{tabular}{|l|l|}
\hline Auswirkung & Kurzdefinition \\
\hline \multirow{4}{*}{ Horizontproblem } & Die verbleibende \\
& Verweildauer der einzelnen \\
& Mitglieder beeinflusst die \\
& Präferenz von langfristigen \\
& Entscheidungen. \\
\hline & Durch die \\
& genossenschaftliche \\
& Institution profitieren alle \\
& Mitglieder, unabhängig \\
Trittbrettfahrerproblem & deren Zeitpunkt des \\
& Eintritts von aktuellen \\
& Leistungen ohne \\
& Berücksichtigung des \\
& individuellen Beitrags. \\
\hline \multirow{5}{*}{ Synergieeffekte } & Produktionsprozesse von \\
& kooperierenden Einheiten \\
& wirken zusammen und \\
& ergeben \\
& Wettbewerbsvorteile. \\
\hline \multirow{5}{*}{ Mitgliederaktivität } & Unterschiedliche \\
& Beteiligung der Mitglieder \\
& im Geschäftsbetrieb einer \\
& Genossenschaft. \\
\hline
\end{tabular}

Quelle: Eigene Ermittlung.

für jüngere Mitglieder attraktiv zu gestalten und mehr einzubinden. Als einen Grund warum es schwer sei Nachwuchs für die Genossenschaft zu motivieren, sei der Konkurrenzdruck der umliegenden Industrie als attraktiver Arbeitgeber, äußerten Interviewpartner A und B. Jedoch wurde deutlich, dass zu einem Großteil die Ehrenämter von den älteren Mitgliedern betreut werden, was der Erfahrung zuzusprechen ist.

Jeder Genossenschaftsführer äußerte, das Heterogenität im Alter in ihrer Genossenschaft zu finden ist. $\mathrm{A}, \mathrm{B}$ und $\mathrm{D}$ berichten von einer guten Zusammenarbeit zwischen den Generationen und Hilfsbereitschaft untereinander. Genossenschaft $\mathrm{C}$ legt dar, dass die jüngeren Mitglieder sich immer weniger mit der Genossenschaft identifizieren und daher kein Interesse zur aktiven Mitarbeit an den Geschäftsabläufen spürbar ist. Zudem betreiben diese den Weinbau im Nebenerwerb oder Hobby, sodass diese nicht wirtschaftlich von der Genossenschaft abhängig sind.
Kein Genossenschaftsvertreter empfand allerdings die Heterogenität im Alter der Mitglieder als ein Problem. Genossenschaft D empfand die Heterogenität im Alter und die Etablierung eines Beirates als eine Chance um eine "Kaderschmiede" für die Zukunft zu etablieren.

\subsection{Berufserfahrung des mitglieds}

Der Ausbildungsgrad im Weinbauhandwerk stellt eine geringe Heterogenität in Genossenschaften dar. Interviewpartner D stellte heraus, dass die jüngeren Generationen zwar häufig besser ausgebildet seien, aber dass dies durch die Erfahrung der Älteren kompensiert wird. Zudem sind alle Betriebe exzellente Traubenlieferanten, betonte Interviewpartner B.

Ein wichtiger Aspekt hingegen ist das wirtschaftliche Verständnis, das zum Teil durch Ausbildung in anderen Berufen erreicht werden kann. Interviewpartner D legte Wert auf eine Diversität in den Gremien. Verschiedene Kompetenzen außerhalb des Weinbaus können einfach durch Nebenerwerbswinzer mit anderen Hauptberufen in die Führungsebene der Genossenschaft eingebracht werden. Allerdings wählt die Generalversammlung die Gremien häufig nur nach der Größe der Weinberge und kleinere Winzer sind weniger engagiert in der Genossenschaft.

Interviewpartner A beschrieb das Nichtwissen und die Unkenntnis vom Markt und das fehlende Wirtschaftsdenken als das größte Hemmnis bei Entscheidungen. Vor allem bei immateriellen Investitionen, wie Markenbildung und -etablierung fehle das Verständnis und der Weitblick.

Dieser und die anderen Genossenschaften beschrieben allerdings, dass das wirtschaftliche Verständnis und Marktaffinität mit der Größe des Betriebes und dessen Führungsaufgaben verbunden ist. Daher wird dies genauer unter Kapitel 4.4 (Art des Mitgliedsbetriebs) behandelt.

\subsection{Ziele des mitglieds}

Die Qualitäts- bzw. Mengenvorstellungen und Unternehmensphilosophien sind von Betrieb zu Betrieb unterschiedlich. Genossenschaften unterstützen und kontrollieren ihre Mitglieder bei Qualitätsvorgaben, dies ist wichtig bei der Etablierung des Portfolios. Eine Genossenschaft produziert nicht nur Premiumweine, so ist die Heterogenität in den Unternehmensphilosophien ein wichtiger Bestandteil der Genossenschaften, um ein passendes Portfolio zu entwerfen. Kleinere Streitereien unter den Mitgliedern in Bezug auf Weinbergsarbeiten kommen zwar vor, diese beeinflussen aber nicht die Entscheidungen der Genossenschaft. Interviewpartner A spricht von einer "sozialen Kontrolle" und "Ächtung" der Kollegen, sodass hier zwar Heterogenität vorhanden ist, dies aber nicht zu Problemen führt.

Interviewpartner $\mathrm{B}$ berichtet von einer Konzentration der Kleinbetriebe auf Premiumprodukte, dies liegt allerdings daran, dass die Großbetriebe häufig keine Kapazitäten für zeitintensive, qualitätsfördernde Maßnahmen im Weinberg haben und "zusehen müssen, dass alle Trauben gesund geentert werden können". Dies hat auch keine negativen Auswirkungen auf die Abläufe der Genossenschaft.

Unterschiedliche Ziele werden in Genossenschaft $\mathrm{C}$ verfolgt, begründet ist dies in der Größe und 
Tabelle 3. Auswirkungen der Heterogenität.

\begin{tabular}{|l|c|c|c|c|}
\hline & $\begin{array}{c}\text { Genossenschaft } \\
\text { A }\end{array}$ & $\begin{array}{c}\text { Genossenschaft } \\
\text { B }\end{array}$ & $\begin{array}{c}\text { Genossenschaft } \\
\text { C }\end{array}$ & $\begin{array}{c}\text { Genossenschaft } \\
\text { D }\end{array}$ \\
\hline Rebfläche & 900 ha & 400 ha & 450 ha & 1400 ha \\
\hline Mitglieder & 1200 & 800 & 800 & 1400 \\
\hline Gründungs jahr & 1900 & 1920 & 1970 & 1970 \\
\hline
\end{tabular}

Die Interviews wurden mit der qualitativen Inhaltsanalyse nach Mayring ausgewertet und die Aussagen der verschiedenen Interviewpartner strukturiert.

Abhängigkeit der Betriebe von der Genossenschaft. Die Existenz der Vollerwerbsbetriebe ist verbunden mit der Performance und Effizienz der Genossenschaft und somit mit dem ausgezahlten Traubengeld. Diese treffen Entscheidungen in der Genossenschaft hinsichtlich Investitionen auf wirtschaftlicher Basis, sodass Trauben in der Genossenschaft effektiver verarbeitet werden können. Für diese Betriebe sind vordergründig die Liquidität und die Höhe des Traubengeldes als Einnahmequelle wichtig. Diese Entscheidungen werden innerhalb des Aufsichtsrates so verabschiedet, da die Vollerwerbsbetriebe diesen bestellen. In der Generalversammlung kommt es anschließend mit den Kleinbetrieben zu Konflikten. Hobby- und Kleinbetriebe wiederum identifizieren sich mit der Genossenschaft nach außen auf Veranstaltungen. Für diese ist die Genossenschaft in erster Linie eine Verkörperung ihrer Interessen auf Weinfesten und sonstigen Veranstaltungen. Diese sehen die Genossenschaft nicht vordergründig, als eine Einnahmequelle und sind nicht an dessen Existenz gebunden. Der Geschäftsführer der Genossenschaft fühlt sich hierbei in der Pflicht auf die Wünsche beider Parteien einzugehen, ist allerdings mehr auf die Wirtschaftlichkeit des Betriebes bedacht und vermittelt auf der Generalversammlung zwischen beiden Interessengruppen. Er sieht dies als Gefahr und Hemmnis bei Entscheidungen.

\subsection{Art des Mitgliedsbetriebes}

Wie bereits bei dem Ausbildungsniveau der Betriebsleiter angesprochen herrscht ein heterogenes wirtschaftliches Verständnis, das auf die Betriebsgröße des Traubenlieferanten zurückzuführen ist.

Genossenschaft A teilt mit, dass die Vertreter des Aufsichtsrats und Vorstands zu 100\% aus Vollerwerbsbetrieben bestehen. Diese Tatsache vereinfacht die Zusammenarbeit des geschäftsführenden Vorstands mit den Gremien. Viele der Vollerwerbsproduzenten führen einen Betrieb mit eigenen Angestellten und sind mit Führungsentscheidungen vertraut. Diese haben zudem eine bessere Bindung zum Markt und betriebswirtschaftliches Verständnis. Hauptkonflikte der Heterogenität in Genossenschaften entstehen, laut Geschäftsführer A, durch ein unterschiedliches Verständnis für den Markt. Dies wird allerdings durch das Vertrauen der Mitglieder in die Führungsebene kompensiert und die kleineren Betriebe folgen den Entscheidungen der größeren Betriebe. Solange die Genossenschaft gesund wirtschaftet und eine, für die Mitglieder angemessene Traubengeldauszahlung, erfolgt, gibt es wenig Konfliktpotential auf Versammlungen.

Interviewpartner B unterstützt diese Aussage, obwohl die Vollerwerbsbetriebe nur einen Anteil von 10\% der Rebfläche bewirtschaften. Jedoch sind die Vollerwerbsbetriebe auch die ausführenden Personen der Ehrenämter.
Dies liegt an der größeren Abhängigkeit und Interesse der Betriebe an der Genossenschaft. Hier sorgen die Entscheidungen der Führungsebene auch für wenige Konflikte, aufgrund des Vertrauens aller Mitglieder in den Führungsstab, dessen "Entscheidungen fast immer von Erfolg gekrönt sind" und diese Mitglieder einer gesunden Genossenschaft sind. Die Entscheidungen, die getroffen werden, beeinflussen allerdings nie die Höhe des Traubengeldes, da Investitionen aus eigenen Mitteln finanziert werden können.

Auch bei Genossenschaft C bildet der Großteil der Gremien die Inhaber der Vollerwerbsbetriebe. Hierbei kommt es allerdings zu Konflikten, die auf die unterschiedlichen Ziele, die in Kapitel 4.2.3 beschrieben wurden, sich auswirken.

In Genossenschaft D bildet die Art der Mitgliedsbetriebe eine (fast) homogene Gruppe. Beinahe 85\% der bewirtschafteten Rebfläche werden von Vollerwerbsbetrieben betreut. Dies erleichtert die Entscheidungsfindung und die wenigen Kleinbetriebe folgen der Meinung der Großbetriebe, so erreicht eine Abstimmung in der Vollversammlung oft eine Zuspruchsquote von $99 \%$.

\subsection{Perspektivische Ausrichtung}

In allen Genossenschaften gibt es Probleme hinsichtlich der perspektivischen Ausrichtung, da in vielen Betrieben eine Nachfolge nicht geklärt ist. Genossenschaft B spricht von einem drohenden Wegfall von bis zu 100 Hektar in den kommenden 10 Jahren aufgrund dieses Problems. Interviewpartner $\mathrm{C}$ berichtet bereits von einem deutlichen Rückgang durch ungesicherte Betriebsnachfolge. Allerdings hat die Tatsache, ob es ein Nachfolger für den Betrieb gibt, keine Auswirkung auf die Entscheidungsfindung der Genossenschaft. Laut Interviewpartner A kompensiert die Identifikation mit der Genossenschaft und der Region, die Tatsache, dass keine Betriebsübergabe geregelt ist. Zudem wird bei den meisten Entscheidungen, das aktuelle Traubengeld nicht beeinflusst. Die unklare Nachfolgeregelung und der einhergehende Wegfall von Rebflächen stellt ein großes Problem für Genossenschaften dar. Die Heterogenität der perspektivischen Ausrichtung der Betriebe beeinflusst allerdings nicht das operative Geschäft der Genossenschaften.

\section{Chancen und probleme der heterogenität}

Heterogenität der Mitglieder ist ein Baustein, auf dem das Geschäftsprinzip der Genossenschaft beruht. Diversität fördert die Möglichkeiten einer Genossenschaft, schafft jedoch auch Konfliktpunkte. Die Unterschiede in den Strukturen von Genossenschaften erfordern 
eine angepasste Herangehensweise mit Heterogenität umzugehen.

Die Altersverteilung wird aufgrund des Strukturwandels und der Konsolidierung von Flächen zwar homogener, jedoch sind in jeder Genossenschaft unterschiedliche Altersgruppen vertreten. Diese arbeiten nicht gegeneinander und verfolgen nicht gänzlich gegenseitige Interessen, sondern profitieren vom der Arbeit miteinander. Genossenschaften versuchen die vorhandenen Jungwinzer in die Führungsebene zu integrieren und Anreize für Neumitglieder zu schaffen. Ein generelles Problem der Landwirtschaft wird der schwindende Nachwuchs und die Alterung der Landwirte bleiben. Der Anteil an passiven Mitgliedern steigt aufgrund der Alterung und der vermehrten Aufgabe der Weinberge, sodass die Mitgliederaktivität sich auf eine Gruppe der aktiven Mitglieder konzentriert. Heterogenität führt zu einer Ansammlung von vielen Kompetenzen in der Genossenschaft. Viele Nebenerwerbsmitglieder besitzen Fähigkeiten die sie in ihren Hauptberufen erwarben und die vielseitig eingesetzt werden können. Eine Aufgabe der Genossenschaft ist es, diese Vorteile zu bündeln und effizient einzusetzen. Dies wirkt sich in einer gesteigerten Mitgliederaktivität aus und ermöglicht das Schöpfen von Synergieeffekten.

Die Ziele der Mitglieder einer Genossenschaft unterscheiden sich, da diese sich unterschiedlich mit der Genossenschaft identifizieren. Die Identifikation der Mitglieder mit der Genossenschaft wird angestrebt, um die Ziele der heterogenen Gruppe zu vereinen und eine gemeinsame strategische Ausrichtung zu verfolgen. Dies kann die Mitgliederaktivität, wie auch das Trittbrettfahrerproblem oder Synergieeffekt fördern oder kompensieren. Welche Ausprägung dies annimmt hängt von dem wirtschaftlichen Erfolg der Genossenschaft ab. Ein Einfluss auf das Horizontproblem konnte nicht festgestellt werden.

Heterogene Mitgliederstrukturen sorgen für ein unterschiedliches Marktverständnis der Genossen. Diese sind zwar alle Betriebsleiter, haben jedoch aufgrund verschiedener Betriebsgrößen einen anderen Bezug zur Wirtschaft und zum Markt. Kleinere Betrieben folgen oft den Meinungen der großen Betriebe, solange der wirtschaftliche Erfolg vorhanden ist und diese der Betriebsführung Vertrauen schenken. Unterschiedliches Marktverständnis kann sich negativ auf das Erreichen von Synergieeffekten beziehen, diese Verbindung wurde bisher nicht berücksichtigt.

Ein großes Problem in landwirtschaftlichen Betrieben ist, dass eine Nachfolge in den Betrieben häufig nicht gesichert ist. In Genossenschaften geht dies mit einem Verlust von Flächen einher, die zur Aufrechterhaltung der Größenvorteile in der Produktion und Vermarktung notwendig sind. Alternative Lösungen, wie der Aufbau von einem eigenen Winzerbetrieb oder die Motivation von Jungwinzern müssen entwickelt werden, um zukünftig am Markt bestehen zu bleiben. Gesetzliche Hürden müssen hierbei überdacht werden und gegebenenfalls angepasst werden, um einen Außenbetrieb der Genossenschaft bei zuführen. Ein negativer Einfluss aufgrund der Heterogenität auf das Horizontproblem ist daher nicht gegeben.

Durch Experteninterviews mit genossenschaftlichen Geschäftsführern konnten die Auswirkungen von Heterogenität in Genossenschaften überprüft werden. Die bestätigten Beziehungen grün - positiv und rot - negativ sind in Abbildung 3 dargestellt.

Abbildung 3: Forschungsmodell - tatsächliche Beziehungen

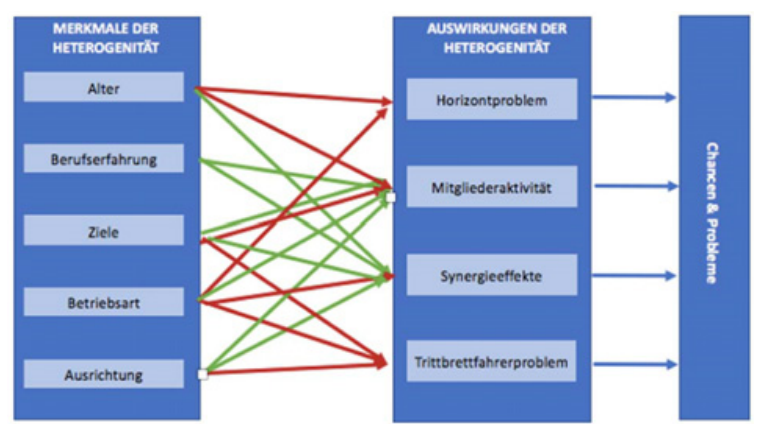

Quelle: Eigene Darstellung

\section{Zusammenfassung}

Der Zweck deutscher Genossenschaften, die Wirtschaftlichkeit ihrer Mitglieder zu fördern, wird durch verschiedene Ausprägung der Heterogenität beeinflusst. Inwiefern die Genossenschaften mit den einzelnen Aspekten der Heterogenität umgehen und nutzen, hängt von der Struktur der Mitglieder und deren Ausprägung ab. Trotz alledem konnten Überschneidungen von Problemen und Chancen der Genossenschaft, wie sie mit Heterogenität umgehen, ermittelt werden. Die größte Herausforderung für eine Genossenschaft bleibt allerdings ihre individuelle Heterogenität zu erkennen und gegenüber ihren Wettbewerbern bestmöglich zu nutzen.

Gipperts Metapher, dass der Genossenschaftsgedanke oft nur präsent sei, solange es allen Mitglieder gut gehe, ist in Hinblick auf die Heterogenität anzupassen: Solange es der Genossenschaft gut gehe, sind Probleme, aufgrund der Heterogenität weniger stark spürbar. Die Ausprägungen dieser machen sich oft nur bemerkbar, wenn der wirtschaftliche Erfolg ausbleibt und nichtmehr gemeinsam an einem Strick gezogen wird. Andererseits kann Heterogenität effizient genutzt werden.

\section{Literaturverzeichnis}

BMJV (2018): Gesetz betreffend die Erwerbs- und Wirtschaftsgenossenschaften. URL.: http://www . gesetze-im-internet.de/geng/__1.html,

Download vom 25. Januar 2018

H. Brügelnann (2002): Heterogenität, Integration und Differenzierung in der Primärstufe. Auflage 1. Band 6. Dezember 2002. VS Verlag für Sozialwissenschaften BWGV (2015): Baden-Württembergische Genossenschaftsverband e.V., Möglichkeiten für Genossenschaften im Weinbau-Strukturwandel, URL.: https://www.wirleben-genossenschaft.de/de/moeglichkeitenfuer-genossenschaften-im-weinbau-

strukturwandel-717.htm, Download vom 7. Februar 2018

DDW (2017): Der deutsche Weinbau, Strukturwandel im Weinbau hält an: Zahl der Betriebe sinkt, Ausgabe 16/2017, S.12

D. Dentoni, D. Menozzi, G.M. Capelli, Group heterogeneity and cooperation on the geographical indication regulation: The case of the "Prosciutto di Parma" 
Consortium, Food Policy 37, Elsevier Ltd, Seiten 207216 (2012)

DGRV, (2018): Historie Genossenschaft. URL: https://www.dgrv.de/de/genossenschaftswesen/ historiege nossenschaft.html, Download vom 26.1.2018

DRV (2017): Zahlen und Fakten. Letzte Aktualisierung 9. Mai 2017, URL.: http://www. raiffeisen.de/presse/zahlen- und-fakten/, Download vom 25. Januar 2018, Darstellung 10.

A. Engels (2002): Genossenschaftliche Kooperationen im Weinbau. Ein Beitrag zur Vereinbarkeit genossenschaftsund unternehmenstheoretischer Erklärungen der Kooperationen. 1.Hrsg. Marburg 2002. Tectum Verlag Marburg

A. Hellberg-Bahr, S. Schlecht, N. Steffen, A. Spiller (2011): Heterogene Mitgliederinteressen in Molkereigenossenschaften - ein Fallbeispiel., Jahrbuch der ÖGA, Band 19 (2), S. 139-148

G. Hendrikse, C. Veerman (2004): On the Future of Cooperatives: Taking Stock, Looking Ahead. In: Hendrikse, G. (Hrsg.), Restructuring Agricultural Cooperatives, Rotterdam: ERIM

J. Höhler (2017): Heterogenität von Mitgliederstrukturen als Herausforderung für Genossenschaften im
Agribusiness. Zeitschrift für das gesamte Genossenschaftswesen. 1/2017

N. Kalogeras J.M.E. Pennings, I.A. van der Lans, P. Garcia, G. van Dijk, Understanding heterogeneous preferences of cooperative members, Agribusiness 25, S. 90-111 (2009)

K. Kricheldorff (2010): Badische Zeitung: Sorge um die Rebflächen - Niederrimsinger Winzergenossenschaft zieht Bilanz. Letzte Aktualisierung 7. Dezember 2010, URL.: http://www.badische-zeitung.de/ breisach/sorge-um-die-rebflaechen--3856, Download vom 25. Januar 2018, S. 1

C. Krieger, J.H. Hanf, V. Belaya (2014): Markenbildung und - führung bei Winzergenossenschaften: Ergebnisse einer Unternehmensbefragung. Berichte über Landwirtschaft, Band 92, Ausgabe 3, Dezember 2014, Herausgegeben vom Bundesministerium für Ernährung und Landwirtschaft

P. Mayring (2010): Qualitative Inhaltsanalyse - Grundlagen und Techniken. 11. Herausgabe. Beltz Verlag M. Minnici, B. Merlin (2016): Fusionen zwischen Winzer - bzw. Weingärtnergenossenschaften in Deutschland - ein Erfolgsmodell?, 18. Internationale Genossenschaftswissenschaftliche Tagung, Luzern, September 2016 